\title{
Robust Fault Detection and Isolation for Stochastic Systems
}

\author{
Jemin George and Irene M. Gregory
}

\begin{abstract}
This paper outlines the formulation of a robust fault detection and isolation scheme that can precisely detect and isolate simultaneous actuator and sensor faults for uncertain linear stochastic systems. The given robust fault detection scheme based on the discontinuous robust observer approach would be able to distinguish between model uncertainties and actuator failures and therefore eliminate the problem of false alarms. Since the proposed approach involves precise reconstruction of sensor faults, it can also be used for sensor fault identification and the reconstruction of true outputs from faulty sensor outputs. Simulation results presented here validate the effectiveness of the robust fault detection and isolation system.
\end{abstract}

\section{INTRODUCTION}

Faults are deviations from the normal behavior of the plant or its instrumentation and they can be categorized into: i) additive process faults, ii) multiplicative process faults, iii) sensor faults, and iv) actuator faults. There exist several fault monitoring procedures which can be used to recognize and distinguish different types of faults [1]. These fault monitoring procedures can be categorized into: i) fault detection, ii) fault isolation, and iii) fault identification. A survey on design methods for fault detection is given in [2]. Most of the existing FDI (fault detection and isolation) schemes are based on measurement residual generation. Generated residual is used to facilitate the decision making procedures involved in FDI. The basic difference between most FDI schemes is the underlying residual generation methods. Few examples of different FDI schemes are the observer based Fault Detection Filters [3]-[5], Kalman filter based Proportional Integral Observers [6], Multiple Model Adaptive Estimators [7], and system identification methods [8].

In this manuscript two types of faults are of concern, i.e., actuator faults and sensor faults. The FDI scheme considered here is the observer based approach. Discontinuous observers such as the sliding mode observers have been successfully used in FDI context [9]. Design of sliding mode observers for detection and reconstruction of actuator and sensor faults is presented in [10] and [11], respectively. The precise reconstruction of faults proposed in [10] assumes the absence of uncertainty. The FDI approaches presented in [11] and [12] introduce an approximate fault reconstruction scheme by minimizing the error between the true fault signal and the reconstructed fault signal. In [13], an FDI scheme for a class of nonlinear uncertain systems is presented by introducing limitations on the structure of the uncertainty. It is important to notice that the precise reconstruction of fault is generally not available in the presence of uncertainty. While the FDI schemes presented in [10]-[13] involves reconstruction of faults, sliding mode observer based FDI approach presented in [14] is based on the measurement residual generation. Though the FDI scheme presented in [14] assumes precise knowledge of system dynamics, a similar FDI scheme which is robust to mismatched uncertainties is presented in [15]. The residual generation presented in [14] and [15] is based on the sliding mode observer scheme where the observer maintain the sliding motion in the presence of mismatched uncertainties, but when fault occurs, the sliding motion is broken and a residual is generated which contains information regarding the fault.

J. George is with Dept. of Mechanical \& Aerospace Engineering, University at Buffalo, SUNY, Buffalo, NY-14260 jgeorge3@buffalo.edu

I. M. Gregory is with NASA Langley Research Center, Hampton, VA-23681 irene.m.gregory@nasa.gov
Though there are numerous literatures on fault detection and identification, very little work is done on developing robust fault detection schemes. Robust actuator/sensor fault detection is a challenging problem due to system modeling errors, external disturbances, and measurement noise. Most of the current fault detection algorithms are model based and they tent to induce false alarms when the plant dynamics differ from the assumed model. This manuscript presents a robust FDI scheme that can precisely detect and isolate simultaneously occurring actuator faults and sensor faults for uncertain linear stochastic systems. The proposed approach is an observer based FDI scheme where a discontinuous observer is used for residual generation. The main highlights of the proposed FDI scheme are

- Multiple actuator and sensor faults are considered

- Proposed technique involves reconstruction of sensor faults and therefore this approach can be used for sensor fault identification and reconstruction of true outputs

- There are no constraints on system uncertainties, both matched and mismatched uncertainties are considered

- Present scheme can be easily extended to nonlinear systems by considering Lipschitz continuous affine nonlinear terms with known Lipschitz constant [13], [15]

The structure of this paper is as follows. A detailed formulation of the observer based FDI scheme is first given. Afterwards, the results from numerical simulations and the concluding remarks are given in sections III and IV, respectively.

\section{OBSERVER-BASED FAult Detection Filter}

Let $\left(\Omega, \mathcal{F},\left\{\mathcal{F}_{t}\right\}_{t \geq t_{0}}, \mathbb{P}\right)$ denotes a complete filtered probability space. Consider an $n^{\text {th }}$-order stochastic system of the following form:

$$
\begin{aligned}
& \dot{\mathbf{X}}_{1}(t)=A_{11} \mathbf{X}_{1}(t)+A_{12} \mathbf{X}_{2}(t)+\mathbf{W}_{1}(t) \\
& \dot{\mathbf{X}}_{2}(t)=A_{21} \mathbf{X}_{1}(t)+A_{22} \mathbf{X}_{2}(t)+B \mathbf{u}(t)+\mathbf{W}_{2}(t) \\
& \mathbf{Y}_{1}(t)=C_{11} \mathbf{X}_{1}(t)+C_{12} \mathbf{X}_{2}(t)+\mathcal{V}_{1}(t) \\
& \mathbf{Y}_{2}(t)=C_{21} \mathbf{X}_{1}(t)+C_{22} \mathbf{X}_{2}(t)+\mathbf{y}_{e}(t)+\mathcal{V}_{2}(t)
\end{aligned}
$$

where $\mathbf{W}_{1}(t)$ and $\mathbf{W}_{2}(t)$ denote stochastic disturbances and $\mathcal{V}_{1}(t)$ and $\mathcal{V}_{2}(t)$ indicate measurement noises. The state vectors, $\mathbf{X}_{1}(t)$ and $\mathbf{X}_{2}(t)$, are of dimensions, $\mathbf{X}_{1}(t) \in \Re^{n-r}$ and $\mathbf{X}_{2}(t) \in \Re^{r}$, respectively. The true state matrices, $A_{11} \in \Re^{(n-r) \times(n-r)}, A_{12} \in$ $\Re^{(n-r) \times r}, A_{21} \in \Re^{r \times(n-r)}, A_{22} \in \Re^{r \times r}$, and the control distribution matrix $B \in \Re^{r \times r}$, are assumed to be unknown. The desired input signal is denoted as $\mathbf{u}_{d}(t)$ and $\mathbf{u}_{e}(t)$ indicates the error in applied control, $\mathbf{u}(t)$, due to actuator faults, i.e.,

$$
\mathbf{u}(t)=\mathbf{u}_{d}(t)+\mathbf{u}_{e}(t)
$$

The stochastic measurement vectors, $\mathbf{Y}_{1}(t)$ and $\mathbf{Y}_{2}(t)$, are of dimensions, $\mathbf{Y}_{1}(t) \in \Re^{m_{1}}$ and $\mathbf{Y}_{2}(t) \in \Re^{m_{2}}$, respectively. The output matrices, $C_{11}, C_{12}, C_{21}$ and $C_{22}$, are assumed to be known. The measurement noise, $\left[\mathcal{V}_{1}^{T}(t) \mathcal{V}_{2}^{T}(t)\right]^{T}=\mathcal{V}(t) \in \Re^{m}$, is assumed to be zero-mean Gaussian white noise process, i.e., $\mathcal{V}(t) \sim \mathcal{N}(\mathbf{0}, R \delta(\tau))$. The vector, $\mathbf{y}_{e}(t)$, indicates sensor failures and is modeled as

$$
\dot{\mathbf{y}}_{e}(t)=\mathbf{f}\left(\mathbf{y}_{e}(t), t\right), \quad \mathbf{y}_{e}\left(t_{0}\right)=\mathbf{0}
$$

where $\mathbf{f}(\cdot)$ is an unknown operator. Stochastic external disturbance $\left[\mathbf{W}_{1}^{T}(t) \mathbf{W}_{2}^{T}(t)\right]^{T}=\mathbf{W}(t) \in \Re^{n}$ is modeled as a linear system driven by a Gaussian white noise process, i.e.,

$$
\dot{\mathbf{W}}(t)=\mathbb{L}(\mathbf{W}(t), t)+\mathcal{W}(t), \quad \mathbf{W}\left(t_{0}\right)=\mathbf{0}
$$


where $\mathbb{L}(\cdot)$ is an unknown linear operator and $\mathcal{W}(t) \in \Re^{n}$, is a zeromean Gaussian white noise process, i.e., $\mathcal{W}(t) \sim \mathcal{N}(\mathbf{0}, \mathcal{Q} \delta(\tau))$.

Assumption 1. Assume the sensor faults are not instantaneous and therefore there exist a known conservative upper bound on $\mathbf{f}\left(\mathbf{y}_{e}(t), t\right)$ such that

$$
\left|\mathbf{f}\left(\mathbf{y}_{e}(t), t\right)\right| \leq \sigma(t), \quad \forall t \geq t_{0}
$$

where $|\cdot|$ denotes the Euclidean norm.

The external disturbance, $\mathbf{W}(t)$, is mean square bounded [16], [17], i.e.

$$
\sup _{t \geq t_{0}} E\left[\mathbf{W}(t) \mathbf{W}^{T}(t)\right] \leq K
$$

where $K$ is a constant matrix whose elements are finite. The assumed (known) model of the plant in (1) is given as

$$
\begin{aligned}
& \dot{\mathbf{x}}_{m_{1}}(t)=A_{m_{11}} \mathbf{x}_{m_{1}}(t)+A_{m_{12}} \mathbf{x}_{m_{2}}(t) \\
& \dot{\mathbf{x}}_{m_{2}}(t)=A_{m_{21}} \mathbf{x}_{m_{1}}(t)+A_{m_{22}} \mathbf{x}_{m_{2}}(t)+B_{m} \mathbf{u}_{d}(t)
\end{aligned}
$$

Define the model-error vectors $\mathcal{D}_{1}(t) \in \Re^{n-r}$ and $\mathcal{D}_{2}(t) \in \Re^{r}$ as

$$
\begin{aligned}
& \mathcal{D}_{1}(t)=\Delta A_{11} \mathbf{X}_{1}(t)+\Delta A_{12} \mathbf{X}_{2}(t)+\mathbf{W}_{1}(t) \\
& \mathcal{D}_{2}(t)=\Delta A_{21} \mathbf{X}_{1}(t)+\Delta A_{22} \mathbf{X}_{2}(t)+\Delta B \mathbf{u}_{d}(t)+\mathbf{W}_{2}(t)
\end{aligned}
$$

where $\Delta A_{11}=A_{11}-A_{m_{11}}, \Delta A_{12}=A_{12}-A_{m_{12}}, \Delta A_{21}=$ $A_{21}-A_{m_{21}}, \Delta A_{22}=A_{22}-A_{m_{22}}$, and $\Delta B=B-B_{m}$.

Assumption 2. Given the system parameter uncertainties are bounded and the system states are bounded in mean square sense, an upper bound on the model error vector $\mathcal{D}(t)$ can be obtained as

$$
\mathbb{P}(|\mathcal{D}(t)| \leq \bar{\mu}(t))=1, \quad \forall t \geq t_{0}
$$

That is, $|\mathcal{D}(t)|$ is almost surely (a.s.) upper bounded by $\bar{\mu}(t)$ for all $t \geq t_{0}$.

Now the plant dynamics in (1) can be written in-terms of known parameters as

$\dot{\mathbf{X}}_{1}(t)=A_{m_{11}} \mathbf{X}_{1}(t)+A_{m_{12}} \mathbf{X}_{2}(t)+\mathcal{D}_{1}(t)$
$\dot{\mathbf{X}}_{2}(t)=A_{m_{21}} \mathbf{X}_{1}(t)+A_{m_{22}} \mathbf{X}_{2}(t)+B_{m} \mathbf{u}_{d}(t)+\mathcal{D}_{2}(t)+B \mathbf{u}_{e}(t)$

Re-parameterize $\mathbf{X}_{2}(t)$ as

$$
\mathbf{X}_{2}(t)=\alpha \mathbf{X}_{2_{\alpha}}(t)+\beta \mathbf{X}_{2_{\beta}}(t)
$$

where $\mathbf{X}_{2_{\alpha}}(t) \in \Re^{r}, \mathbf{X}_{2_{\beta}}(t) \in \Re^{r}, \alpha$ and $\beta$ are user selected scalar parameters. Now $\dot{\mathbf{X}}_{2}(t)$ can be written as $\dot{\mathbf{X}}_{2}(t)=\alpha \dot{\mathbf{X}}_{2_{\alpha}}(t)+$ $\beta \dot{\mathbf{X}}_{2_{\beta}}(t)$. Select $\dot{\mathbf{X}}_{2_{\alpha}}(t)$ and $\dot{\mathbf{X}}_{2_{\beta}}(t)$ as

$$
\begin{aligned}
& \dot{\mathbf{X}}_{2_{\alpha}}(t)=\frac{1}{\alpha} A_{m_{21}} \mathbf{X}_{1}(t)+A_{m_{22}} \mathbf{X}_{2_{\alpha}}(t)+\frac{1}{\alpha} \mathcal{D}_{2}(t) \\
& \dot{\mathbf{X}}_{2_{\beta}}(t)=A_{m_{22}} \mathbf{X}_{2_{\beta}}(t)+\frac{1}{\beta} B_{m} \mathbf{u}_{d}(t)+\frac{1}{\beta} B \mathbf{u}_{e}(t)
\end{aligned}
$$

Remark 1. One of the main challenges in the design of observer based FDI scheme is the presence of coupled system uncertainties and actuator faults [15]. In the presence of coupled system uncertainties and actuator faults, it is difficult to design an observer that yields measurement residual which is only sensitive to the actuator faults. Notice that the re-parametrization of $\mathbf{X}_{2}(t)$ given in (7) allows decoupling of system uncertainties and actuator faults as shown in (8).

Assumption 3. Assume there exists a bounded vector $\zeta(t) \in \Re^{r}$ such that $B \mathbf{u}_{e}(t)=B_{m} \boldsymbol{\zeta}(t)$, i.e.,

$$
\boldsymbol{\zeta}(t)=B_{m}^{-1} B \mathbf{u}_{e}(t) \text { and }|\boldsymbol{\zeta}(t)| \leq \xi(t), \quad \forall t \geq t_{0}
$$

After appending the sensor error dynamics given in (3), the extended system can be written as

$$
\begin{aligned}
{\left[\begin{array}{c}
\dot{\mathbf{X}}_{1}(t) \\
\dot{\mathbf{X}}_{2_{\alpha}}(t) \\
\dot{\mathbf{X}}_{2_{\beta}}(t) \\
\dot{\mathbf{y}}_{e}(t)
\end{array}\right] } & =\left[\begin{array}{cccc}
A_{m_{11}} & \alpha A_{m_{12}} & \beta A_{m_{12}} & 0 \\
\frac{1}{\alpha} A_{m_{21}} & A_{m_{22}} & 0 & 0 \\
0 & 0 & A_{m_{22}} & 0 \\
0 & 0 & 0 & A_{\mathbf{y}_{e}}
\end{array}\right]\left[\begin{array}{c}
\mathbf{X}_{1}(t) \\
\mathbf{X}_{2_{\alpha}}(t) \\
\mathbf{X}_{2_{\beta}}(t) \\
\mathbf{y}_{e}(t)
\end{array}\right] \\
+ & {\left[\begin{array}{c}
0 \\
0 \\
\frac{1}{\beta} B_{m} \\
0
\end{array}\right] \mathbf{u}_{d}(t)+\left[\begin{array}{cccc}
I & 0 & 0 & 0 \\
0 & \frac{1}{\alpha} I & 0 & 0 \\
0 & 0 & \frac{1}{\beta} B_{m} & 0 \\
0 & 0 & 0 & I
\end{array}\right]\left[\begin{array}{c}
\mathcal{D}_{1}(t) \\
\mathcal{D}_{2}(t) \\
\boldsymbol{\zeta}(t) \\
\mathbf{h}(\cdot)
\end{array}\right] }
\end{aligned}
$$

where $\mathbf{h}(\cdot)=\mathbf{f}(\cdot)-A_{\mathbf{y}_{e}} \mathbf{y}_{e}$ and $A_{\mathbf{y}_{e}} \in \Re^{m_{2} \times m_{2}}$ is a user selected Hurwitz matrix. Let $\mathbf{Z}(t)=\left[\begin{array}{llll}\mathbf{X}_{1}^{T}(t) & \mathbf{X}_{2_{\alpha}}^{T}(t) & \mathbf{X}_{2_{\beta}}^{T}(t) & \mathbf{y}_{e}^{T}(t)\end{array}\right]^{T}$, now the above extended system can be rewritten as

$$
\begin{aligned}
\dot{\mathbf{Z}}(t)=F \mathbf{Z}(t)+G_{3} \mathbf{u}_{d}(t)+G_{1} \mathcal{D}_{1}(t)+G_{2} \mathcal{D}_{2}(t) & +G_{3} \zeta(t) \\
& +G_{4} \mathbf{h}(\cdot)
\end{aligned}
$$

where $F=\left[\begin{array}{cccc}A_{m_{11}} & \alpha A_{m_{12}} & \beta A_{m_{12}} & 0 \\ \frac{1}{\alpha} A_{m_{21}} & A_{m_{22}} & 0 & 0 \\ 0 & 0 & A_{m_{22}} & 0 \\ 0 & 0 & 0 & A_{\mathbf{y}_{e}}\end{array}\right]$ and

$$
G \triangleq\left[\begin{array}{llll}
G_{1} & G_{2} & G_{3} & G_{4}
\end{array}\right]=\left[\begin{array}{cccc}
I & 0 & 0 & 0 \\
0 & \frac{1}{\alpha} I & 0 & 0 \\
0 & 0 & \frac{1}{\beta} B_{m} & 0 \\
0 & 0 & 0 & I
\end{array}\right]
$$

Let $H=\left[\begin{array}{llll}C_{11} & \alpha C_{12} & \beta C_{12} & 0 \\ C_{21} & \alpha C_{22} & \beta C_{22} & I\end{array}\right]$, the measurement equations can be rewritten as $\mathbf{Y}(t)=H \mathbf{Z}(t)+\mathcal{V}(t)$. Now the system in (1) can be written as the following dynamically equivalent form

$$
\begin{aligned}
\dot{\mathbf{Z}}(t)=F \mathbf{Z}(t)+G_{3} \mathbf{u}_{d}(t)+ & G_{1} \mathcal{D}_{1}(t)+G_{2} \mathcal{D}_{2}(t) \\
& +G_{3} \boldsymbol{\zeta}(t)+G_{4} \mathbf{h}(\cdot) \\
\mathbf{Y}(t)=H \mathbf{Z}(t)+\mathcal{V}(t) &
\end{aligned}
$$

Remark 2. Even though the above representation of the plant is a nonminimal realization, the observability of the extended system may be obtained by making appropriate changes to the state matrix, $F$, and the corresponding changes to $\mathcal{D}_{1}(t), \mathcal{D}_{2}(t)$, and $\mathbf{h}(\cdot)$.

Consider the following partition of $G_{1}$ as $n-r$ column vectors, $G_{2}$ as $r$ column vectors, $G_{3}$ as $r$ column vectors, and $G_{4}$ as $m_{2}$ column vectors as shown below

$$
\begin{aligned}
G_{1} & =\left[\begin{array}{llll}
\mathbf{g}_{11} & \mathbf{g}_{12} & \ldots & \mathbf{g}_{1(n-r)}
\end{array}\right] \\
G_{2} & =\left[\begin{array}{llll}
\mathbf{g}_{21} & \mathbf{g}_{22} & \ldots & \mathbf{g}_{2 r}
\end{array}\right] \\
G_{3} & =\left[\begin{array}{llll}
\mathbf{g}_{31} & \mathbf{g}_{32} & \ldots & \mathbf{g}_{3 r}
\end{array}\right] \\
G_{4} & =\left[\begin{array}{llll}
\mathbf{g}_{41} & \mathbf{g}_{42} & \ldots & \mathbf{g}_{4 m_{2}}
\end{array}\right]
\end{aligned}
$$

Also consider the individual elements of the vectors $\zeta(t), \mathcal{D}(t)$, and $\mathbf{h}(\cdot)$, i.e.,

$$
\boldsymbol{\zeta}(t)=\left[\begin{array}{c}
\zeta_{1}(t) \\
\vdots \\
\zeta_{r}(t)
\end{array}\right], \quad \mathcal{D}(t)=\left[\begin{array}{c}
\mathcal{D}_{1}(t) \\
\vdots \\
\mathcal{D}_{n}(t)
\end{array}\right] \quad \text { and } \quad \mathbf{h}(\cdot)=\left[\begin{array}{c}
h_{1}(\cdot) \\
\vdots \\
h_{m_{2}}(\cdot)
\end{array}\right]
$$

Now the extended system in (9) can be written in summation form as

$$
\begin{aligned}
\dot{\mathbf{Z}}(t)= & F \mathbf{Z}(t)+\sum_{i=1}^{n-r} \mathbf{g}_{1 i} \mathcal{D}_{i}(t)+\sum_{j=1}^{r} \mathbf{g}_{2 j} \mathcal{D}_{n-r+j}(t) \\
& +\sum_{k=1}^{r} \mathbf{g}_{3 k} \zeta_{k}(t)+\sum_{l=1}^{m_{2}} \mathbf{g}_{4 l} h_{l}(\cdot)+G_{3} \mathbf{u}_{d}(t)
\end{aligned}
$$


Define $\mathcal{G}_{1} \triangleq\left[\begin{array}{ll}G_{1} & G_{2}\end{array}\right], \mathcal{G}_{2} \triangleq\left[\begin{array}{ll}G_{3} & G_{4}\end{array}\right]$, and $\boldsymbol{\eta}^{T}(t) \triangleq$ $\left[\begin{array}{ll}\boldsymbol{\zeta}^{T}(t) & \mathbf{h}^{T}(\cdot)\end{array}\right]^{T}$. Now (11) may be rewritten as

$$
\dot{\mathbf{Z}}(t)=F \mathbf{Z}(t)+G_{3} \mathbf{u}_{d}(t)+\sum_{i=1}^{n} \mathcal{G}_{1 i} \mathcal{D}_{i}(t)+\sum_{j=1}^{r+m_{2}} \mathcal{G}_{2 j} \eta_{j}(t)
$$

where $\mathcal{G}_{1 k}$ and $\mathcal{G}_{2 k}$ are the $k^{\text {th }}$ column vectors of $\mathcal{G}_{1}$ and $\mathcal{G}_{2}$ matrices, respectively. Now $\ell=1,2, \ldots, r+1$ observers of the following from are considered

$$
\begin{aligned}
& \text { If } \ell \leq r \\
& \begin{aligned}
\dot{\mathbf{Z}}^{\ell}(t)= & F \widehat{\mathbf{Z}}^{\ell}(t)+L^{\ell}\left[\mathbf{Y}(t)-H \widehat{\mathbf{Z}}^{\ell}(t)\right]+G_{3} \mathbf{u}_{d}(t)+ \\
& \sum_{i=1}^{n} \mathcal{G}_{1 i} \mu_{i}^{\ell}(t)+\sum_{j=1}^{\ell-1} \mathcal{G}_{2 j} \nu_{j}^{\ell}(t)+\sum_{j=\ell+1}^{r+m_{2}} \mathcal{G}_{2 j} \nu_{j}^{\ell}(t)
\end{aligned} \\
& \quad \text { If } \ell=r+1 \\
& \dot{\widehat{\mathbf{Z}}}^{\ell}(t)=F \widehat{\mathbf{Z}}^{\ell}(t)+L^{\ell}\left[\mathbf{Y}(t)-H \widehat{\mathbf{Z}}^{\ell}(t)\right]+G_{3} \mathbf{u}_{d}(t)+ \\
& \qquad \sum_{i=1}^{n} \mathcal{G}_{1 i} \mu_{i}^{\ell}(t)+\sum_{j=1}^{r+m_{2}} \mathcal{G}_{2 j} \nu_{j}^{\ell}(t)
\end{aligned}
$$

where $L^{\ell} \in \Re^{\left(n+r+m_{2}\right) \times m}$ is the observer gain corresponding to the $\ell^{\text {th }}$ observer. The observer inputs are denoted as, $\left[\begin{array}{lll}\nu_{1}^{\ell}(t) & \ldots & \nu_{\left(r+m_{2}\right)}^{\ell}(t)\end{array}\right]^{T} \triangleq \nu^{\ell}(t) \in \Re^{r+m_{2}}$, and

$$
\left[\begin{array}{lll}
\mu_{1}^{\ell}(t) & \ldots & \mu_{n}^{\ell}(t)
\end{array}\right]^{T} \triangleq \mu^{\ell}(t) \in \Re^{n}, \forall \ell=1,2, \ldots, r+1
$$

Equations (13) and (14) correspond to the typical observer model. The observer gain $L^{\ell}$ and the observer inputs $\boldsymbol{\mu}^{\ell}(t)$ and $\boldsymbol{\nu}^{\ell}(t)$ corresponding to the $\ell^{\text {th }}$ observer are selected so that the generated residual obtained from observers given in (13) is asymptotically stable if there is no fault in the $\ell^{\text {th }}$ actuator and the residual obtained from the observer given in (14) is asymptotically stable despite any actuator or sensor fault occurrences.

Define the observer error as $\widetilde{\mathbf{Z}}^{\ell}(t)=\mathbf{Z}(t)-\widehat{\mathbf{Z}}^{\ell}(t)$. After subtracting (14) from (11), the observer error dynamics can be written as

$$
\begin{aligned}
& \dot{\overrightarrow{\mathbf{Z}}}^{\ell}(t)=\left[F-L^{\ell} H\right] \widetilde{\mathbf{Z}}^{\ell}(t)-L^{\ell} \mathcal{V}(t)+ \\
& \sum_{i=1}^{n} \mathcal{G}_{1 i}\left[\mathcal{D}_{i}(t)-\mu_{i}^{\ell}(t)\right]+\sum_{j=1}^{r+m_{2}} \mathcal{G}_{2 j}\left[\eta_{j}(t)-\nu_{j}^{\ell}(t)\right]
\end{aligned}
$$

It is important to note that the solution to the stochastic differential equation given in (15) cannot be based on the ordinary mean square calculus because the integral involved in the solution depends on $\mathcal{V}(t)$, which is of unbounded variation, i.e., $E\left[\mathcal{V}(t) \mathcal{V}^{T}(t+\tau)\right]=$ $R \delta(\tau)$. For the treatment of this class of problems, the stochastic differential equation can be rewritten in Itô form as [18], [19]

$$
\begin{aligned}
d \widetilde{\mathbf{Z}}^{\ell}(t)=\{ & {\left[F-L^{\ell} H\right] \widetilde{\mathbf{Z}}^{\ell}(t)+\sum_{i=1}^{n} \mathcal{G}_{1 i}\left[\mathcal{D}_{i}(t)-\mu_{i}^{\ell}(t)\right]+} \\
& \left.\sum_{j=1}^{r+m_{2}} \mathcal{G}_{2 j}\left[\eta_{j}(t)-\nu_{j}^{\ell}(t)\right]\right\} d t-L^{\ell} d \mathcal{B}(t)
\end{aligned}
$$

where the zero-mean Gaussian white noise $\mathcal{V}(t)$ is written as the increments of stationary Wiener process with zero-mean and the correlation of increments

$$
E\left[\{\mathcal{B}(\tau)-\mathcal{B}(\zeta)\}\{\mathcal{B}(\tau)-\mathcal{B}(\zeta)\}^{T}\right]=R|\tau-\zeta|
$$

Details on stochastic Itô calculus can be found in [19]. The observer error corresponding to the $\ell^{\text {th }}$ observer, $\widetilde{\mathbf{Z}}^{\ell}(t)$, is a stochastic process and therefore the stability of the observer error dynamics given in (16) is depicted either as moment stability or stability in probabilistic sense. The stability in probabilistic sense is usually known as almost sure (a.s.) stability and it is defined as follows [16]:

Definition 1. The stochastic process $\widetilde{\mathbf{Z}}^{\ell}(t)$ is asymptotically stable with probability 1 , or almost surely asymptotically stable, if

$$
\mathbb{P}\left(\widetilde{\mathbf{Z}}^{\ell}(t) \rightarrow \mathbf{0} \quad \text { as } \quad t \rightarrow \infty\right)=1
$$

Notice that the almost sure stability of the observer error is impossible due to the persistently acting measurement noise $\mathcal{B}(t)$. Therefore it is desirable for the observer error corresponding to the $\ell^{\text {th }}$ observer, $\widetilde{\mathbf{Z}}^{\ell}(t)$, to have a dynamics that follows

$$
d \widetilde{\mathbf{Z}}_{m}^{\ell}(t)=\left\{\left[F-L^{\ell} H\right] \widetilde{\mathbf{Z}}_{m}^{\ell}(t)\right\} d t+L^{\ell} d \mathcal{B}(t)
$$

Let $\widetilde{\mathcal{Z}}^{\ell}(t)=\widetilde{\mathbf{Z}}^{\ell}(t)-\widetilde{\mathbf{Z}}_{m}^{\ell}(t)$, now subtracting (18) from (16) yields

$$
\begin{aligned}
d \widetilde{\mathcal{Z}}^{\ell}(t)=\{ & {\left[F-L^{\ell} H\right] \widetilde{\mathcal{Z}}^{\ell}(t)+\sum_{i=1}^{n} \mathcal{G}_{1 i}\left[\mathcal{D}_{i}(t)-\mu_{i}^{\ell}(t)\right] } \\
& \left.+\sum_{j=1}^{r+m_{2}} \mathcal{G}_{2 j}\left[\eta_{j}(t)-\nu_{j}^{\ell}(t)\right]\right\} d t
\end{aligned}
$$

Given next is an approach for the selection of the observer gain $L^{\ell}$ and the observer inputs $\boldsymbol{\mu}^{\ell}(t)$ and $\boldsymbol{\nu}^{\ell}(t)$ corresponding to the $\ell^{\text {th }}$ observer based on the stochastic Lyapunov approach.

Since the only information regarding the true observer error is in the form of measurement residual, one do not have full access to the signal $\widetilde{\mathcal{Z}}^{\ell}(t)$, i.e., one only has access to $\widetilde{\mathcal{Y}}^{\ell}(t)=H \widetilde{\mathcal{Z}}^{\ell}(t)$. Based on (19), $d \widetilde{\mathcal{Y}}^{\ell}(t)$ can be written as

$$
\begin{aligned}
& d \widetilde{\mathcal{Y}}^{\ell}(t)=\left\{H\left[F-L^{\ell} H\right] \widetilde{\mathcal{Z}}^{\ell}(t)+\right. \\
& \left.\sum_{i=1}^{n} H \mathcal{G}_{1 i}\left[\mathcal{D}_{i}(t)-\mu_{i}^{\ell}(t)\right]+\sum_{j=1}^{r+m_{2}} H \mathcal{G}_{2 j}\left[\eta_{j}(t)-\nu_{j}^{\ell}(t)\right]\right\} d t
\end{aligned}
$$

Based on Assumptions 1 and 3, define an upper bound on $\boldsymbol{\eta}(t)$ as

$$
|\boldsymbol{\eta}(t)| \leq \bar{\nu}(t), \quad \forall t \geq t_{0}
$$

Theorem 1. Given the Assumptions 1, 2, and 3, the individual fault detection filters given in Eq. (13) guarantee almost sure asymptotic stability of $\widetilde{\mathcal{Y}}^{\ell}(t)$ if there is no fault occurrence in the $\ell^{\text {th }}$ actuator and the fault detection filter given in Eq. (14) guarantees almost sure asymptotic stability of $\widetilde{\mathcal{Y}}^{r+1}(t)$ despite any actuator or sensor fault occurrences, if the observer gain $L^{\ell}$ corresponding to the $\ell^{\text {th }}$ observer is selected so that the following matrix Lyapunov inequality is satisfied

$$
\begin{aligned}
{\left[F-L^{\ell} H\right]^{T} } & H^{T} H P^{\ell} H^{T} H+ \\
& H^{T} H P^{\ell} H^{T} H\left[F-L^{\ell} H\right]+Q^{\ell} \leq 0
\end{aligned}
$$

and the observer inputs corresponding to the $\ell^{\text {th }}$ observer are selected as

$$
\begin{array}{r}
\mu_{i}^{\ell}(t)=\operatorname{sgn}\left\{\left(\tilde{\mathcal{Y}}^{\ell}(t)\right)^{T} H P^{\ell} H^{T} H \mathcal{G}_{1 i}\right\} \bar{\mu}(t), \\
\forall i=1, \ldots, n \\
\nu_{j}^{\ell}(t)=\operatorname{sgn}\left\{\left(\tilde{\mathcal{Y}}^{\ell}(t)\right)^{T} H P^{\ell} H^{T} H \mathcal{G}_{2 i}\right\} \bar{\nu}(t), \\
\forall j=1, \ldots, r+m_{2}
\end{array}
$$




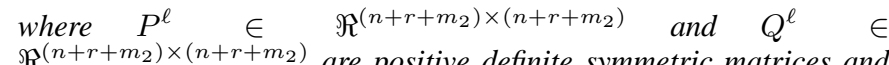
$\Re^{\left(n+r+m_{2}\right) \times\left(n+r+m_{2}\right)}$ are positive definite symmetric matrices and $\operatorname{sgn}\{\cdot\}$ denotes the signum function or the sign function.

Proof: Construct a Lyapunov function candidate of the form $V\left(\widetilde{\mathcal{Y}}^{\ell}(t)\right)=\left(\widetilde{\mathcal{Y}}^{\ell}(t)\right)^{T} H P^{\ell} H^{T} \widetilde{\mathcal{Y}}^{\ell}(t)$. Now using the Itô formula [19], $d V\left(\widetilde{\mathcal{Y}}^{\ell}(t)\right)$ can be calculated as

$$
\begin{gathered}
d V\left(\tilde{\mathcal{Y}}^{\ell}(t)\right)=\left\{( \tilde { \mathcal { Y } } ^ { \ell } ( t ) ) ^ { T } \left\{\left[F-L^{\ell} H\right]^{T} H^{T} H P^{\ell} H^{T} H+\right.\right. \\
\left.H^{T} H P^{\ell} H^{T} H\left[F-L^{\ell} H\right]\right\} \widetilde{\mathcal{Y}}^{\ell}(t) \\
+2\left(\widetilde{\mathcal{Y}}^{\ell}(t)\right)^{T} H P^{\ell} H^{T} H \sum_{i=1}^{n} \mathcal{G}_{1 i}\left[\mathcal{D}_{i}(t)-\mu_{i}^{\ell}(t)\right] \\
\left.+2\left(\widetilde{\mathcal{Y}}^{\ell}(t)\right)^{T} H P^{\ell} H^{T} H \sum_{j=1}^{r+m_{2}} \mathcal{G}_{2 j}\left[\eta_{j}(t)-\nu_{j}^{\ell}(t)\right]\right\} d t
\end{gathered}
$$

After substituting (20), $\mathfrak{L} V\left(\tilde{\boldsymbol{y}}^{\ell}\right)$ can be written as

$$
\begin{aligned}
\mathfrak{L} V\left(\tilde{\boldsymbol{y}}^{\ell}\right) & \leq-\left(\widetilde{\boldsymbol{y}}^{\ell}(t)\right)^{T} Q^{\ell} \widetilde{\boldsymbol{y}}^{\ell}(t) \\
& +2 \sum_{i=1}^{n}\left(\widetilde{\boldsymbol{y}}^{\ell}(t)\right)^{T} H P^{\ell} H^{T} H \mathcal{G}_{1 i}\left[\mathcal{D}_{i}(t)-\mu_{i}^{\ell}(t)\right] \\
& +2 \sum_{j=1}^{r+m_{2}}\left(\widetilde{\boldsymbol{y}}^{\ell}(t)\right)^{T} H P^{\ell} H^{T} H \mathcal{G}_{2 j}\left[\eta_{j}(t)-\nu_{j}^{\ell}(t)\right]
\end{aligned}
$$

where the operator $\mathfrak{L}\{\cdot\}$ acting on $V(\mathbf{x}, t)$ is defined as

$$
\mathfrak{L} V(\mathbf{x}, t)=\lim _{d t \rightarrow 0} \frac{1}{d t} E[d V(\mathbf{X}(t), t) \mid \mathbf{X}(t)=\mathbf{x}]
$$

Substituting (21) and (22) yields

$$
\begin{aligned}
\mathfrak{L} V\left(\tilde{\boldsymbol{y}}^{\ell}\right) \leq- & \left(\widetilde{\boldsymbol{y}}^{\ell}(t)\right)^{T} Q^{\ell} \widetilde{\boldsymbol{y}}^{\ell}(t)+ \\
& 2 \sum_{i=1}^{n}\left\{\left(\widetilde{\boldsymbol{y}}^{\ell}(t)\right)^{T} H P^{\ell} H^{T} H \mathcal{G}_{1 i} \mathcal{D}_{i}(t)-\right. \\
& \left.\left|\left(\widetilde{\boldsymbol{y}}^{\ell}(t)\right)^{T} H P^{\ell} H^{T} H \mathcal{G}_{1 i}\right| \bar{\mu}(t)\right\}+ \\
& 2 \sum_{j=1}^{r+m_{2}}\left\{\left(\widetilde{\boldsymbol{y}}^{\ell}(t)\right)^{T} H P^{\ell} H^{T} H \mathcal{G}_{2 j} \eta_{j}(t)-\right. \\
& \left.\left|\left(\widetilde{\boldsymbol{y}}^{\ell}(t)\right)^{T} H P^{\ell} H^{T} H \mathcal{G}_{2 j}\right| \bar{\nu}(t)\right\}
\end{aligned}
$$

Thus

$$
\mathfrak{L} V\left(\tilde{\boldsymbol{y}}^{\ell}(t)\right) \leq-\left(\tilde{\boldsymbol{y}}^{\ell}(t)\right)^{T} Q^{\ell} \tilde{\boldsymbol{y}}^{\ell}(t)
$$

Therefore the $\ell=(r+1)^{\text {th }}$ observer in (14) is almost surely asymptotically stable despite the occurrence of any actuator or sensor faults. Based on the given proof one could easily make the argument that if there is no fault occurrence in the $\ell^{\text {th }}$ actuator, where $1 \leq \ell \leq r$, then the $\ell^{\text {th }}$ observer given in (13) is almost surely asymptotically stable. Thus any observed residual $\widetilde{\boldsymbol{y}}^{\ell}(t)$, will indicate a fault occurrence in the $\ell^{\text {th }}$ actuator.

Any observed residual in the $\ell^{\text {th }}$ observer given in (13), where $1 \leq \ell \leq r$, indicates a fault occurrence in the $\ell^{\text {th }}$ actuator. Based on the observability condition one could easily show that the estimated or the observer generated sensor error terms, $\hat{\mathbf{y}}_{e}(t)$, asymptotically approaches the true sensor error, $\mathbf{y}_{e}(t)$. Therefore $\hat{\mathbf{y}}_{e}(t)$ obtained from the observer given in (14) can be directly used for sensor fault detection. That is, if $\hat{\mathbf{y}}_{e}(t)=\mathbf{0}$, then there is no sensor fault and if $\hat{y}_{e_{i}}(t) \neq 0$, then the nonzero $\hat{y}_{e_{i}}(t)$ indicates a fault occurrence in the $i^{\text {th }}$ sensor. Moreover, by subtracting $\hat{\mathbf{y}}_{e}(t)$ from the measured output yields the true system output.

\section{Numerical Simulations}

Numerical simulation results are presented in this section to validate the efficiency of the proposed FDI scheme. Consider a stochastic system of the form given in (1) where the true system matrices are given as

$$
\begin{aligned}
& A_{11}=\left[\begin{array}{ll}
0 & 0 \\
0 & 0
\end{array}\right], \quad A_{12}=\left[\begin{array}{ll}
1 & 0 \\
0 & 1
\end{array}\right], \quad A_{21}=\left[\begin{array}{cc}
-1.3 & 0.01 \\
-0.12 & -1.8
\end{array}\right], \\
& A_{22}=\left[\begin{array}{cc}
-0.9 & -0.011 \\
0.3 & -3.4
\end{array}\right], \quad B=\left[\begin{array}{cc}
2.4 & -0.23 \\
-0.11 & 1.5
\end{array}\right]
\end{aligned}
$$

and the assumed system matrices are

$$
\begin{aligned}
& A_{m_{11}}=\left[\begin{array}{ll}
0 & 0 \\
0 & 0
\end{array}\right], \quad A_{m_{12}}=\left[\begin{array}{ll}
1 & 0 \\
0 & 1
\end{array}\right], \quad B_{m}=\left[\begin{array}{cc}
3.19 & -0.31 \\
-0.1 & 2.01
\end{array}\right] \\
& A_{m_{21}}=\left[\begin{array}{cc}
-1.13 & 0 \\
0 & -1.18
\end{array}\right], \quad A_{m_{22}}=\left[\begin{array}{cc}
-0.99 & 0 \\
0 & -2.98
\end{array}\right]
\end{aligned}
$$

The system output matrices are given as $C_{11}=$ $\left[\begin{array}{cc}I_{2 \times 2} & 0_{2 \times 2}\end{array}\right]^{T}, C_{12}=\left[\begin{array}{ll}0_{2 \times 2} & I_{2 \times 2}\end{array}\right]^{T}, C_{21}=$ $\left[\begin{array}{cc}0.95 & 1.7 \\ -2.4 & 1.54\end{array}\right], C_{22}=\left[\begin{array}{cc}0.43 & -2.31 \\ 1.3 & 0.43\end{array}\right]$. For simulation purposes, the external disturbance is modeled as $\mathbf{W}_{1}=\mathbf{0}$ and $\mathbf{W}_{2}(t)=\left[\begin{array}{lll}W_{21}(t) & W_{22}(t)\end{array}\right]^{T}$ is given as

$$
\begin{aligned}
& W_{21}(t)=-W_{21}(t)+\mathcal{W}_{1}(t) \\
& W_{22}(t)=-W_{22}(t)+\mathcal{W}_{2}(t)
\end{aligned}
$$

where $\left[\mathcal{W}_{1}(t) \mathcal{W}_{2}(t)\right]^{T}=\mathcal{W}(t)$ is zero-mean Gaussian white noise process with

$$
E\left[\mathcal{W}(t) \mathcal{W}^{T}(t+\tau)\right]=10^{-2} \times I_{2 \times 2} \delta(\tau)
$$

The measurement noise, $\mathcal{V}(t) \triangleq \mathcal{V}(t) \in \mathbb{R}^{6}$, is assumed to be zeromean Gaussian white noise process with

$$
E\left[\mathcal{V}(t) \mathcal{V}^{T}(t+\tau)\right]=10^{-2} \times I_{6 \times 6} \delta(\tau)
$$

Note that $\mathcal{D}_{1}=\mathbf{0}$ and $\mathcal{D}_{2}(t)$ is given as

$$
\mathcal{D}_{2}(t)=\Delta A_{21} \mathbf{X}_{1}(t)+\Delta A_{22} \mathbf{X}_{2}(t)+\Delta B \mathbf{u}_{d}(t)+\mathbf{W}_{2}(t)
$$

where the desired control input $\mathbf{u}_{d}(t)$ is given in Fig. 1. For the re-parametrization of the system states, the constants $\alpha$ and $\beta$ are selected as $\alpha=\beta=1$. Note that the two possible sensors faults are

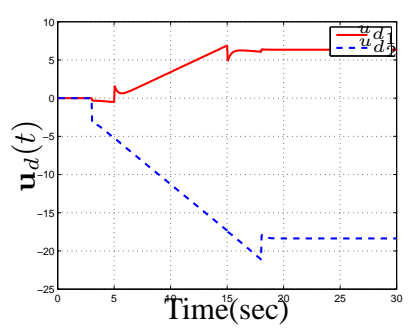

Fig. 1. Desired Control Input

associated with the fifth and sixth outputs. Two fault scenarios are considered here and the details on the fault scenarios are 
1) Fault Scenario I: For the first fault scenario, the faults are associated with the second actuator and the fifth output sensor. The actuator fault occurs at thirty seconds $(\mathrm{sec})$ and the sensor fault occurs at sixty-five sec. Given in Fig. 2 are the $\mathbf{u}_{e}(t)$ and $\mathbf{y}_{e}(t)$ corresponding to the first fault scenario.

2) Fault Scenario II: For the second fault scenario considered, it is assumed that the faults are associated with the first actuator, the fifth and the sixth output sensors. The fault associated with the first actuator occurs at thirty sec. The fifth and sixth output sensor faults occur at sixty-five sec and eighty-five sec, respectively. Given in Fig. 3 are the $\mathbf{u}_{e}(t)$ and $\mathbf{y}_{e}(t)$ corresponding to the second fault scenario.

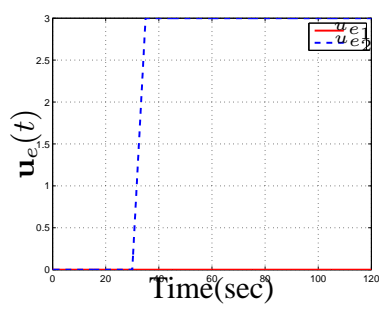

(a) Actuator Faults

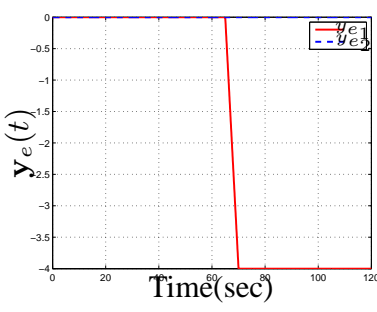

(b) Sensor Faults
Fig. 2. Fault Scenario I

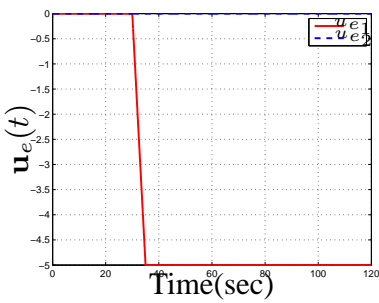

(a) Actuator Faults

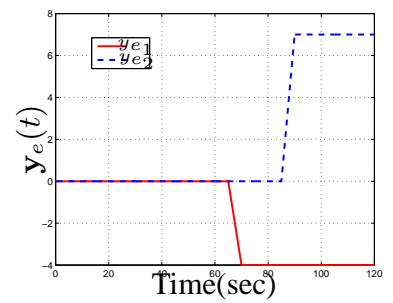

(b) Sensor Faults
Fig. 3. Fault Scenario II

Note that for the system considered here, there are two actuators and therefore three different observers are designed. For both fault scenarios considered, $A_{y}$ is selected as $A_{y}=0_{2 \times 2}, P^{\ell}$ is selected as $P^{\ell}=10^{-2} \times I, \forall \ell \in\{1,2,3\}$. The observer gain is calculated as $L^{1}=L^{2}=L^{3}=$

$\left[\begin{array}{cccccc}49.9299 & -0.2986 & -0.2402 & 0.7390 & -2.5331 & -0.3157 \\ 0.3753 & 49.8876 & -0.3033 & -0.2856 & 0.7403 & -1.7170 \\ 0.1847 & 0.0195 & 24.9291 & -0.3715 & 1.0916 & -0.7319 \\ -0.3075 & 0.3204 & 0.5508 & 24.6784 & 1.2925 & 1.9531 \\ 0.1847 & 0.0195 & 24.9291 & -0.3715 & 1.0916 & -0.7319 \\ -0.3075 & 0.3204 & 0.5508 & 24.6784 & 1.2925 & 1.9531 \\ -47.3614 & -85.1196 & -21.7003 & 115.5758 & 49.6501 & -0.7843 \\ 120.0819 & -76.9340 & -64.9756 & -21.7627 & 0.8362 & 49.8110\end{array}\right]$

The extended output matrix $H$ and the matrix $G$ can be calculated as

$$
H=\left[\begin{array}{llll}
C_{11} & C_{12} & C_{12} & 0_{4 \times 2} \\
C_{21} & C_{22} & C_{22} & I_{2 \times 2}
\end{array}\right], \quad G=\left[\begin{array}{ccc}
0_{2 \times 2} & 0_{2 \times 2} & 0_{2 \times 2} \\
I_{2 \times 2} & 0_{2 \times 2} & 0_{2 \times 2} \\
0_{2 \times 2} & B_{m} & 0_{2 \times 2} \\
0_{2 \times 2} & 0_{2 \times 2} & I_{2 \times 2}
\end{array}\right]
$$

Details on the results obtained for both fault scenarios are given next.

1) Fault Scenario I: Given in Fig. 4 are the true error vectors, $\mathcal{D}_{2}(t)=\Delta A_{21} \mathbf{X}_{1}(t)+\Delta A_{22} \mathbf{X}_{2}(t)+\Delta B \mathbf{u}_{d}(t)+\mathbf{W}_{2}(t), \boldsymbol{\zeta}(t)=$ $B_{m}^{-1} B \mathbf{u}_{e}(t)$, and $\mathbf{h}(\cdot)=\mathbf{f}(\cdot)$ corresponding to the first fault scenario.
For simulation purposes the upper bounds on $\mathcal{D}_{2}(t), \boldsymbol{\zeta}(t)$, and $\mathbf{h}(\cdot)$ are selected as

$$
\begin{aligned}
\left|\mathcal{D}_{2_{1}}(t)\right| & \leq 10 & \left|\mathcal{D}_{2_{2}}(t)\right| & \leq 20 \\
\left|\zeta_{1}(t)\right| & \leq 4 & \left|\zeta_{2}(t)\right| & \leq 3 \\
\left|h_{1}(t)\right| & \leq 2 & \left|h_{2}(t)\right| & \leq 2
\end{aligned}
$$

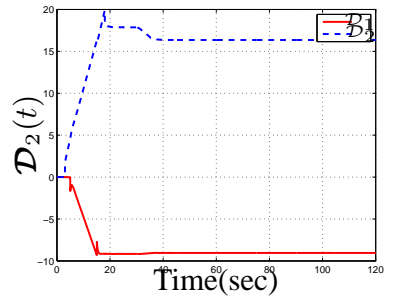

(a) Model Error

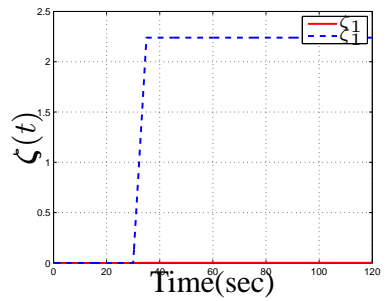

(b) Input Error

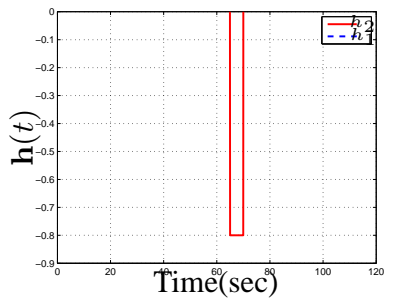

(c) Output Error Rates

Fig. 4. Fault Scenario I: Error Vectors

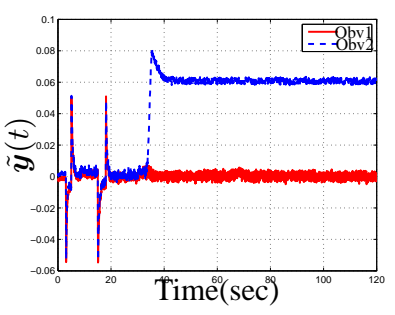

(a) Residual

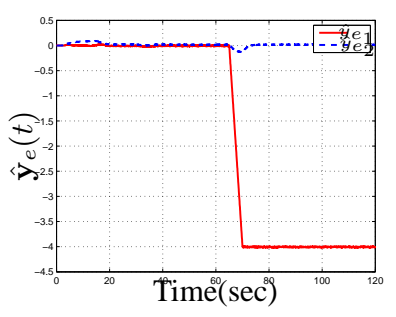

(b) Estimated Sensor Faults
Fig. 5. Fault Scenario I: Observer Residual and Estimated Sensor Faults

Given in Fig. 5 are the generated residual and the estimated sensor errors corresponding to the first fault scenario. Figure 5(a) contains the measurement residual generated for observer one and two. The first two kinks in the residual are due to the start of input application that occurs around five seconds and the leveling-off the input to its steady state value around twenty seconds. Notice the jump in observer two residual around thirty seconds due to the fault occurrence in the second actuator. Figure 5(b) contains the estimated sensor errors obtained from the third observer. Note that the estimated sensor error is similar to the true sensor error given in Fig. 2(b).

2) Fault Scenario II: Given in Fig. 6 are the error vectors, $\mathcal{D}_{2}(t), \boldsymbol{\zeta}(t)$, and $\mathbf{h}(\cdot)$ corresponding to the second fault scenario. The upper bounds on error vectors used here are the same upper bounds used for the first fault scenario.

Given in Fig. 7 are the generated residual and the estimated sensor errors corresponding to the second fault scenario. Figure 7(a) contains the measurement residual generated for observer one and two. Notice the sudden increase in observer one residual around thirty seconds due to the fault occurrence in the first actuator. Figure 7(b) contains the estimated sensor errors obtained from the third observer. Note that the estimated sensor errors are similar to the true sensor errors given in Fig. 3(b). 


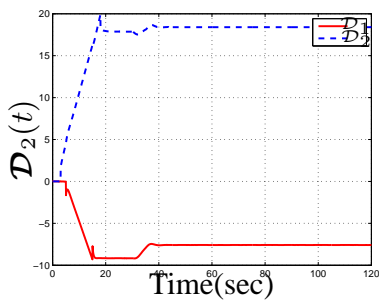

(a) Model Error

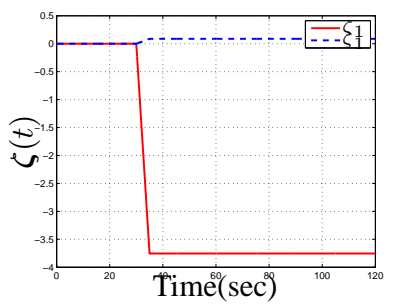

(b) Input Error

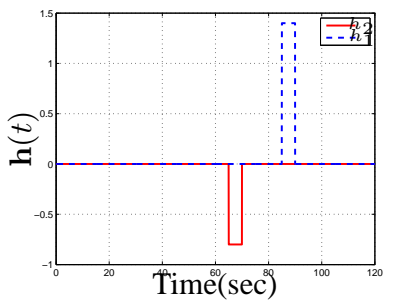

(c) Output Error Rates

Fig. 6. Fault Scenario II: Error Vectors

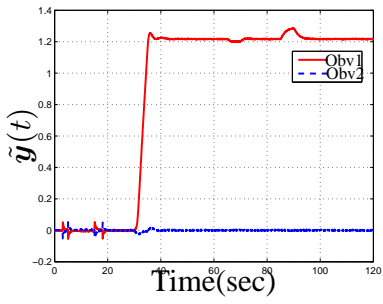

(a) Residual

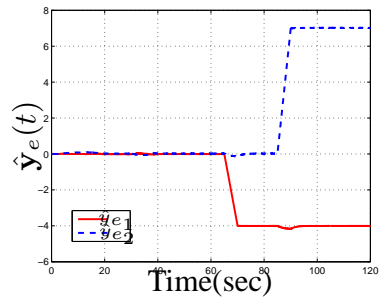

(b) Estimated Sensor Faults
Fig. 7. Fault Scenario II: Observer Residual and Estimated Sensor Faults

\section{CONCLUSION}

Robust actuator/sensor fault detection is a challenging problem due to the effects of modeling errors, system process noise, and measurement noise. This manuscript outlines the formulation of a robust fault detection and isolation scheme that can precisely detect and isolate simultaneously occurring actuator faults and sensor faults for uncertain linear stochastic systems. The given robust fault detection scheme would be able to distinguish between model uncertainties and actuator failure and therefore eliminate the problem of false alarms. The presented approach involves precise reconstruction of sensor faults and therefore this approach can be used for sensor fault identification and the reconstruction of true outputs from faulty sensor outputs. The proposed approach is an observer based fault detection and isolation scheme where a discontinuous observer is used for residual generation.

The proposed approach assume conservative upper bounds on the system uncertainties, the actuator faults, and the sensor fault rates. A bank of discontinuous observers is designed for fault detection and isolation scheme where the number of observers is based on the number of actuators. The observer gain and the discontinuous observer inputs are selected so that the observed residual is almost surely asymptotically stable if there is no actuator fault occurrence. As a result, any observed residual would indicate a fault occurrence in the corresponding actuator. In addition to the bank of observers designed for actuator fault detection, a robust discontinuous observer is designed so that the estimated or the observer generated sensor error terms asymptotically approaches the true sensor error. Therefore, the sensor error estimates obtained from the robust observer can be directly used for sensor fault detection, isolation and identification. Moreover, by subtracting the estimated sensor errors from the measured outputs, true system outputs can be generated. The simulation results reveal clear indication of actuator faults despite the presence of matched system uncertainties and external disturbances. Moreover, the estimated sensor errors are identical to the true sensor error regardless of the measurement noise present.

\section{REFERENCES}

[1] J. Gertler, Fault Detection and Diagnosis in Engineering Systems. New York: Marcel Dekker, 1998.

[2] A. Willsky, "A survey of design methods for failure detection in dynamic systems," Automatica, vol. 12, no. 6, pp. 601-611, November 1976.

[3] R. V. Beard, "Failure accommodation in linear systems through selfreorganization," Ph.D. Thesis, Massachusetts Institute of Technology, 1971.

[4] H. L. Jones, "Failure detection in linear systems," Ph.D. Thesis, Massachusetts Institute of Technology, 1973.

[5] R. L. Chen, "Failure detection filters for robust analytical redundancy," Ph.D. Thesis, University of California, Los Angeles, 2000.

[6] B. Shafai, C. Pi, and S. Nork, "Robust fault detection using proportional integral observers," Proceedings of the 5th Biannual World Automation Congress, vol. 14, pp. 515-520, 2002.

[7] T. Menke and P. Maybeck, "Sensor/actuator failure detection in the vista f- 16 by multiple model adaptive estimation," Aerospace and Electronic Systems, IEEE Transactions on, vol. 31, no. 4, pp. 1218-1229, October 1995.

[8] R. Isermann, "Process fault detection based on modeling and estimation methods: A survey," Automatica, vol. 20, no. 4, pp. 387-404, July 1984.

[9] C. Edwards, S. K. Spurgeon, and R. J. Patton, "Sliding mode observers for fault detection and isolation," Automatica, vol. 36, no. 4, pp. 541553, April 2000.

[10] C. P. Tan and C. Edwards, "Sliding mode observers for detection and reconstruction of sensor faults," Automatica, vol. 38, no. 10, pp. 18151821, October 2002.

[11] — "Sliding mode observers for robust detection and reconstruction of actuator and sensor faults," International Journal of Robust and Nonlinear Control, vol. 13, no. 5, pp. 443-463, April 2003.

[12] B. Jiang, M. Staroswiecki, and V. Cocquempot, "Fault estimation in nonlinear uncertain systems using robust sliding-mode observers," Control Theory and Applications, IEE Proceedings of, vol. 151, no. 1, pp. 29-37, January 2004.

[13] X.-G. Yan and C. Edwards, "Robust sliding mode observer-based actuator fault detection and isolation for a class of nonlinear systems," International Journal of Systems Science, vol. 39, no. 4, pp. 349-359, April 2008.

[14] W. Chen and M. Saif, "A sliding mode observer-based strategy for fault detection, isolation, and estimation in a class of lipschitz nonlinear systems," International Journal of Systems Science, vol. 38, no. 12, pp. 943-955, January 2007.

[15] _ - "Observer-based strategies for actuator fault detection, isolation, and estimation in a class of nonlinear systems," Control Theory \& Applications, IET, vol. 38, no. 12, pp. 943-955, January 2007.

[16] H. J. Kushner, Stochastic Stability and Control. New York, NY: Academic Press, 1967, ch. 2.

[17] T. T. Soong, Random Differential Equations in Science and Engineering. New York, NY: Academic Press, 1973, ch. 4-5.

[18] T. T. Soong and M. Grigoriu, Random Vibration of Mechanical and Structural Systems. Englewood Cliffs, NJ: Prentice Hall, 1993, ch. 4.

[19] M. Grigoriu, Stochastic Calculus. Boston, MA: Birkhäuser, 2002, ch. $1-5$. 